\title{
Die Geographischen Institutionen in der Schweiz
}

Die Zusammenhänge und das Zusammenwirken der verschiedenen geographischen Gesellschaften und Institute mit der Schweizerischen Geographischen Gesellschaft (SGgG) und der Schweizerischen Geographischen Kommission (SGgK) lassen sich wie folgt umschreiben: Die Schweizerische Geographische Gesellschaft ist die Dachgesellschaft der Fachgesellschaften und der Regionalgesellschaften. Sie steht in Verbindung zur International Geographical Union (IGU).

Die Schweizerische Geographische Kommission vereinigt die Vertreter der Hochschulinstitute sowie der Fachgesellschaften. Sie ist die Verbindungsstelle zur Schweizerischen Naturforschenden Gesellschaft (SNG).

Um eine Uebersicht zu geben, haben die SGgG und SGgK ein Organigramm (Abb. 1) und ein Organisationsstatut aufgestellt.

Organisationsstatut

Schweiz. Geographische Gesellschaft Geographische Kommission der SNG

1. Die Schweizerische Geographische Gesellschaft (SGgG) vereinigt die schweizerischen Geographen als Einzelmitglieder, sowie Zweig-, d.h. Fach- und regional-lokale Gesellschaften, als Kollektivmitglieder: nämlich die Schweizerische Geomorphologische Gesellschaft (SGMG), die Schweizerische Gesellschaft für Angewandte Geographie (SGAG), den Verein Schweizerischer Geographielehrer (VSGg), die Schweizerische Gesellschaft für Kartographie (SGK), die GeographischEthnologische Gesellschaft Basel, die Geographische Gesellschaft Bern, die Société de Géographie de Genève, die Société Neuchâteloise de Géographie, die Ostschweizerische Gesellschaft und die Geographisch-Ethnographische Gesellschaft Zürich.

Mit Veranstaltungen und Publikationen fördert sie die geographische Wissenschaft. Insbesondere gibt sie zusammen mit der Geographisch-Ethnographischen Gesellschaft Zürich die Vierteljahresschrift "Geographica Helve- tica" heraus. Sie unterstützt des weitern die Raumplanung und den Umweltschutz und wirkt bei der Lösung von Umweltaufgaben mit. Auf nationaler Ebene und als Mitglied der Schweizerischen Naturforschenden Gesellschaft pflegt sie die Beziehungen zu Nachbarwissenschaften und deren Organisationen. Auf internationaler Ebene vertritt sie die Schweiz in der IGU; der Zentralvorstand bildet zugleich deren Schweizerisches Nationalkomitee.

2. Die Geographische Kommission (GgK) bildet ein Organ der Schweizerischen Naturforschenden Gesellschaft (SNG) . Sie fasst die Vertreter der geographischen Wissenschaft, namentlich der geographischen Hochschulinstitute, zusammen und unterstützt koordinierend deren Tätigkeit. Ihre Mitglieder können sich an den Sitzungen durch andere Angehörige ihres Instituts resp. Fachbereichs vertreten lassen.

Die SGgG und die GgK haben die Möglichkeit, spezielle Anliegen durch gemeinsame Arbeitsgruppen bearbeiten $\mathrm{zu}$ lassen. Diese Arbeitsgruppen sind gegenüber dem erweiterten Vorstand (E.V.) der SGgG und der GgK verantwortlich. Ihre Präsidenten laden zu den Sitzungen ein, über welche Protokolle zu führen sind; diese gehen an die Mitglieder der betreffenden Arbeitsgruppe sowie, zum Zweck der Orientierung, an die Präsidenten der SGgG und der GgK. Die Ergebnisse von Vernehmlassungen sind im Prinzip dem Präsidenten der SGgG zuzuleiten, der sie weitervermittelt.

3. Oberstes Organ der SGgG ist die Hauptversammlung, ihr untergeordnet der E.V., dem u.a. die Delegierten der Zweiggesellschaften und der Redaktor der "Geographica Helvetica" angehören; beide treten mindestens je einmal jährlich zusammen. Die Hauptversammlung wählt auf die Dauer von vier Jahren den Präsi- 


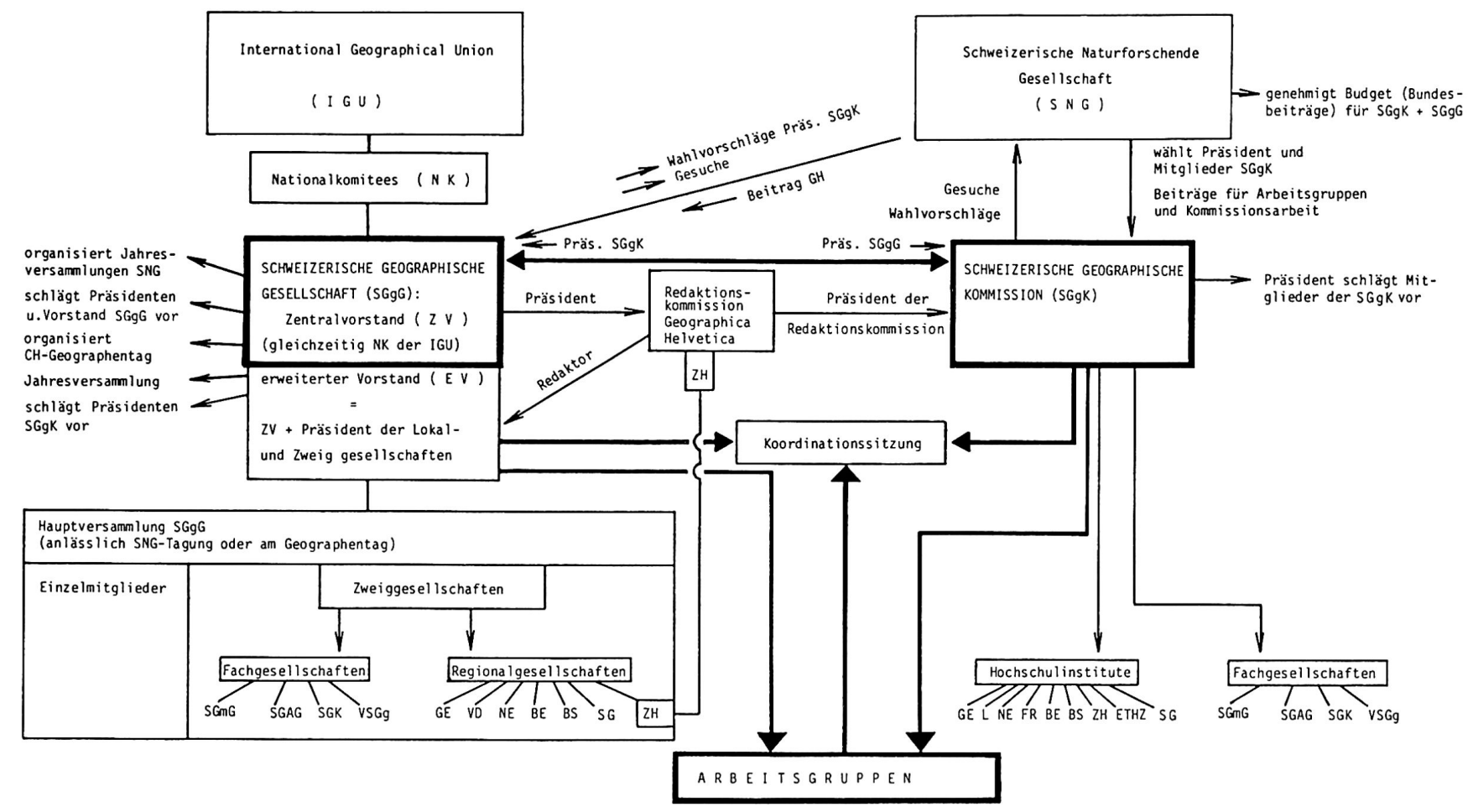


denten - der abtretende Zentralvorstand unterbreitet dafür einen Vorschlag - sowie die übrigen Mitglieder des Zentralvorstandes, die vom künftigen Präsidenten vorgeschlagen werden. Der Zentralvorstand soll regional gebildet werden und im wechsel die verschiedenen Landesteile und Fachrichtungen angemessen erfassen. Dabei soll, um die Kontinuität der Arbeit zu gewährleisten, die $\mathrm{Ab}-$ lösung der Mitglieder nach Möglichkeit gestaffelt erfolgen.

4. Der Präsident der GgK wird auf Vorschlag des E.V. der SGgG durch den Senat der SNG gewählt. Ebenso wählt der Senat der SNG die ihm vom jeweiligen Präsidenten der GgK vorgeschlagenen übrigen Mitglieder der Kommission. In deren Zusammensetzung soll eine gewisse Kontinuität vorherrschen.

5. Der Zentralvorstand der SGgG und die GgK sollen sich in der Weise ergänzen, dass ihre Zusammensetzung insgesamt ausgeglichen ist. Zwischen beiden Gremien hat enges Einvernehmen $\mathrm{zu}$ bestehen; ex officio soll jeder der beiden Präsidenten oder sein Stellvertreter auch den Sitzungen der andern Organisation beiwohnen. Ziel ist ein intensives Parallelwirken namentlich in wissenschaftlicher Beziehung.

Einmal jährlich treten, auf Einladung des Präsidenten der SGgG, der E.V. der SGgG und die GgK zu einer Koordinationssitzung zusammen.

6. Eng soll auch der Kontakt mit der Zeitschrift "Geographica Helvetica" sein. Der Präsident von deren Redaktionskommission gehört ex officio der der GgK, der Redaktor dem E.V. der SGgG, und der Präsident der SGgG der Redaktionskommission der "Geographica Helvetica" an.

Bern, 31. März 1984

Der Präsident der

Schweizerischen Geographischen Gesellschaft

Dr. Erich Schwabe

$--$

Der Präsident der Schweizerischen Geographischen Komm ssion

Prof. Dr. Klaus Aerni 